\title{
BIOSYSTEMATICS AND EVOLUTIONARY NOISE
}

\author{
W. H. Wagner Jr. *
}

\section{Summary}

The main pillars of plant evolution are the populations of nomial species - diploid, sexual, and outbreeding. These basic stocks represent the diversity which has resulted from millions of years of phylogenetic change. Hybrids have occasionally appeared, but most have been sterile or ill-adapted. Polyploids, apomicts, inbreeders, and hybrids are common today and we have no reason to believe that they did not occur even among early land plants. The fact, however, that normal species with normal life cycles still prevail today strongly indicates that such plants have always been responsible for the primary thrust of evolution. A kind of evolutionary noise is produced through the repeated origin of populations with deleteriously modified life cycles in which the advantages of diploidy, sexuality, outbreeding, and species purity are counteracted or cancelled. Such deviations persist for longer or shorter periods while the normal parental stock continues to evolve.

After decades of alleged "biosystematic" studies, we may inquire just how much real contribution has been made to our understanding of plant evolution. Have we concentrated on important questions of divergence of populations into species, genera, and so on; or have we been carried away with side branches and blind alleys that go nowhere. In particular, how much genuine innovation in evolution have polyploidy, inbreeding, apomixis, and hybridization provided? How much of what we studied was merely evolutionary noise?

Divergence is the essential feature of evolution, and it is caused by mutation, recombination, and natural selection on the population level. Our central job as systematists is to investigate organismic relationships and to find explanations for them. A major goal is to determine affinities between the perhaps 300,000 species of land plants worth recognizing on the earth, their classification and their phylogeny. We are concerned with the principal clusters of diversity: subspecies, species, genera, etc., the basic pathways of change: ancestors, major divergences, character trends, and the causes for such changes, the role of selection and adaptation. The vast amount of trivial variation which occurs between individuals and populations of plant species appears to be more or less random, and the systematist endeavors to discover meaningful clusters and patterns. Plant systematics is a very broad biological field which correlates its data from chemistry, cytology, anatomy, morphology, ecology, geology, and geography (Constance, 1964; Wagner, 1968a).

Paradoxically, the bulk of what has been referred to since the time of W. Camp (1943) as "biosystematics" has been in fact a narrow field of study. "Biosystematics" or "experimental taxonomy" has been primarily preoccupied with such subjects as chromosome numbers (especially aneuploidy, polyploidy), chromosome pairing behaviour, upsets in the life cycle such as sterility and apomixis, and interspecific and intergeneric hybrids. Top items in the proposed "Biosystematic Data Storage Bank" are chromosome numbers and hybrids. The content of the present symposium on "Biosystematics at the Cross-roads" illustrates this point. Models for research in so-called "biosystematics" have beer based on such organisms as fruitflies, corn, wheat, hawksbeard, and tobacco.

There has been increasing feeling in recent years that classical "biosystematics" has reached its plateau and that, indeed, very little that is new has appeared in recent

* Matthaei Botanical Gardens, University of Michigan, Ann Arbor, Mich., U.S.A. 
years. In the 40 's and 50 's, however, there was still tremendous enthusiasm for chromosome studies. The chromosomes were believed to be of much more value than other systematic characters (e.g., morphology) because they contained the genes. In this period we emphasized the wheat story - a spectacular case of amphidiploidy AABBDD. Homologous chromosomes paired only with homologous chromosomes, and species and genera were so different genetically that they lacked the gene-by-gene homology required for the pairing reaction to work. ${ }^{1}$ Each species ideally had a constant chromosome number, and some authors argued that if two populations should differ in chromosome number, even if they were morphologically alike, they should be treated as separate species. There were two kinds of "species" - "taxonomic" and "biological".

Our ideas about these matters have changed, or are being changed, especially now that we increasingly realize that observations of chromosomes may not reveal the true genetic situation at all. It is even possible that much of what we see of chromosomes under the light microscope may be trivial or even irrelevant to the real genetics of the organism.

Do polyploidy, apomixis, inbreeding, and hybridization actually cause or promote anything in evolution? For practically every case that seems to show some correlation, we detect another that seems to show the opposite. Is polyploidy correlated with cold? Aridity? Insularity? Weediness? Does aneuploidy itself lead to speciation? (Cf. Stebbins, 1950; Lewis, 1969). Why are there so many species and genera that remain safely euploid which undergo extensive evolution and speciation? Do apomicts have narrower or broader ranges than their sexual progenitors? Are they more uniform or variable than their sexual relatives? Do hybrids commonly, rarely, or ever produce new lines of evolution? Do hybrids yield new characters or merely blends of old? (Cf. Stebbins, 1969; Wagner, 1968b; 1969).

As we find more and more exceptions to our ideas, the exceptions may come to equal or exceed the non-excepticns. More and more species are being found to have both polyploid sectors and apomictic sectors. The chromosome data may not fit with the other data (cf. Wagner, 1963). Where breeding tests are used, distantly related taxa may hybridize and closely related ones not (e.g., Mirov, 1967). We now realize that causes for failure of crossing may be numerous and diverse, and failure to find or make a particular hybrid is not necessarily evidence that the experimental parents are too different genetically and phylogenetically to cross.

It is, without doubt, fitting in science that we do find questions with many answers and generalizations with many exceptions. What we are concerned with here, however, is a matter of what is important, a judgement of value. It is timely that we attempt to gain a broad perspective, especially in view of some of the recent discoveries in genetics. In connection with chromosome numbers, Walter H. Lewis has discovered in the common American spring beauty, Claytonia, no less than four dozen different cytotypes in plants which otherwise a taxonomist would place in a single taxon. If chromosome number can be so variable and still yield essentially the same product, what are we to think? In connection with chromosome pairing, wheat researchers (Riley and Law, 1965) have demonstrated simple genes in wheat which control the pairing of homeologous genomes. How much of pairing behavior that we have reported in the past is merely a result of simple chromoscme compatibility factors?

I have wandered far from the subject of evolution at this point and am discussing merely vagaries in the chromosome apparatus. Significant evolution calls for changes more profound than these - changes in physiology, morphology, and ecology. We

1 It is interesting to note that today we still do not understand the causes of chromosome pairing. nor, in fact, what is the ultimate relationship beween genes and chromosomes. 
have probably given too much attention to the packaging of the genetic material as chomosomes and too little attention to the genetic material itself. If we can generalize from our present knowledge of chromosomes, I shall not be surprised that upon more search we will find thousands upon thousands of new examples of aneuploids, polyploids, inbreeders, apomicts, and interspecific hybrids. How much will they add to our knowledge? Our understanding? Will they contribute to our basic comprehension of the systematic and evolutionary relationships of plants? In the broad picture of evolution, may these phenomena not merely be trivial aberrations and defects in the biology of plants, produced by simple genetic transformations or chance fertilizations, which plants can tolerate, but which have little or no importance to the big picture?

I am reminded of a lecture I gave some years ago on "evolution" involving polyploidy and hybridization. After the lecture was over, a comparative morphologist asked the question: "Is this really evolution?" At the time I thought he was teasing, but the remark bothered me, and I believe now that I understand what he really meant. The point is this: On the earth today, after perhaps 450 millions of years of evolution on the land, the majority of vascular plant stocks (the main branches in Fig. 1) are diploid, sexual, and at least partially outbreeding. Furthermore, the populations of most closely allied species and subspecies are allopatric and separated from their nearest relatives. If polyploidy, apomixis, inbreeding, and interspecific hybridization had, of themselves, truly led to new lines of evolution and possessed significant selective advantages, they would long since have pre-empted the primitive conditions. We cannot imagine that the core of evolution from the earliest times involved other than such basic lines of species as are shown in Fig. 1, i.e. diploid, sexual, and outbreeding. Deviations from the latter have been secondary to the primary thrust of evolution.

From what we know of these phenomena in all parts of the earth today we can expect polyploids, apomicts, inbreeders, and hybrids to arise anywhere in the picture. The same species may possess polyploid forms, apomictic forms, and even obligately inbreeding forms. Interspecific and intergeneric hybrids will arise from time to time to blur the picture by producing intermediates which blunt the effects of evolutionary divergence.

If plants of the past were anything like those of the present, we have no reason to suppose that the situation, at least in higher plants, was ever much different. Polyploids must have continuously arisen from diploids, apomicts from sexuals, inbreeders from outbreeders, and hybrids from divergent species, all of these deviations in the normal flow of evolution tending to interfere with further specializations involving other biological traits. These more or less random upsets in the life cycle and normal interbreeding within populations of species were merely disturbances in the on-going course of evolutionary differentiation. Presumably, in time each of the deviations would die out; but others became generated to replace them. If a diploid could become polyploid once, it probably could do so again; if two species could form one hybrid, they could probably form another.

These phenomena all influence in one way or another the role of the life cycle in evolution. Take the fern, as an example, and its familiar stages: sporangium, meiosis, spore, gametophyte, gametangia, gametes, syngamy, embryo, and young sporophyte (Foster and Gifford, 1959). Each stage plays its own part in evolutionary divergence and adaptation to the habitat. Homologous life cycles are found in all other groups of land plants as well, and in each, the whole is needed to accomplish the two fundamental needs of the dynamic population - the requirements of dispersal and establishment and the facilitation of evolutionary change and adaptation. Any number of defects may mar the intricate perfection of this process. The taxon may exist as a sporophyte only, and reproduce entirely by vegetative means (as is characteristic of the bulk of pteridophytic hybrids). Or (in ferns, at least) it may exist only as a gametophyte, as 
is true of several tropical fern families which are represented in continental North America by species that grow as far north as Virginia and Indiana in the east (Farrar, 1967) and Alaska in the west (Taylor, 1967). In other defective life cycles, only mitospores may be formed by the sporophyte, and the gametophyte simply produces new sporophytes directly by vegetative proliferation, as shown by A. M. Evans (1964). In such extreme cases, where the life cycle deletes both meiosis and syngamy, further evolutionary adaptation is presumably cut off. When such defects arose in plants of the past they ultimately became extinct, because the modification of the life cycle was such that normal capacities for change were destroyed.

How does polyploidy relate to the life cycle? This is a controversial question, in part because amphidiploids are regarded by many as normal species, no different in nature from diploid species, and in part because we are not certain in all cases whether higher numbers per se arose necessarily by multiplication of genomes - the higher numbers may be primitive (e.g., in ferns, where $2 \mathrm{n}$ numbers between ca. 40 and 120 are common to all orders and families). If we accept the classical idea of polyploidy, i.e., that homologous or homeologous alleles are reduplicated in fours, sixes, eights, and so on, then the chances of expression of new mutations becomes sharply limited. The meiotic and syngametic processes in the life cycle thus lose their efficacy in permitting change. "The effect of polyploidy on the genetic system is to put a strong damper on the evolutionary process" (Mosquin, 1966). On theoretical grounds at least, and given equal selective forces, the capacity for evolution of tetraploids falls behind diploids, of octoploids still further behind, and so on. Decaploids and duodecaploids are rare and probably always have been, but this is not necessarily due to mechanical difficulties of having too many chromosomes as one might assume; in the adder'stongue fern genus, Ophioglossum, we observe 2 n numbers of over 1200 chromosomes, but meiosis and spore production seem unimpaired and the life cycle is evidently normal.

How does inbreeding relate to the life cycle? Obviously, if the ability to outbreed is lost completely, then the rate of recombination accomplished by meiosis and syngamy will become greatly reduced. New mutations are prevented from spreading through the population, and the functional significance of the life cycle with respect to promoting evolution is largely lost.

If a given taxon happens to be an interspecific hybrid, it will most likely be sterile. If it spreads at all in its habitat, this will be accomplished probably by vegetative means. If the chromosome number doubles, the hybrid may become an amphidiploid, but then the presumed limitations of polyploidy will be generated. In the rare cases that two well differentiated species happen to be interfertile enough to produce fertile progeny, their hybrids will usually have to fit into some hybrid niche. Such fertile hybrids will therefore tend to be transient, disappearing once the differentiated community returns and the parental species re-occupy their normal habitats.

How, then, can we visualize the broad picture of plant evolution? The main pillars are now, and probably always have been, populations of normal species - diploid, sexual, and outbreeding. The diversity which has resulted from millions of years of phylogenetic change is represented by these basic stocks. Hybrids have occasionally appeared, but most have been sterile or ill-adapted. Polyploids, apomicts, inbreeders, and hybrids are common today and we have no reason to believe that they did not occur even among early land plants. The fact, however, that normal species with normal life cycles still prevail today strongly indicates that such plants have always been responsible for the primary thrust of evolution. Assuming at least some uniformitarianism in the plant biosphere, and accepting today's picture as a guide to what has been, I suggest that the picture of phylogeny is like that shown in Fig. 1. A kind 


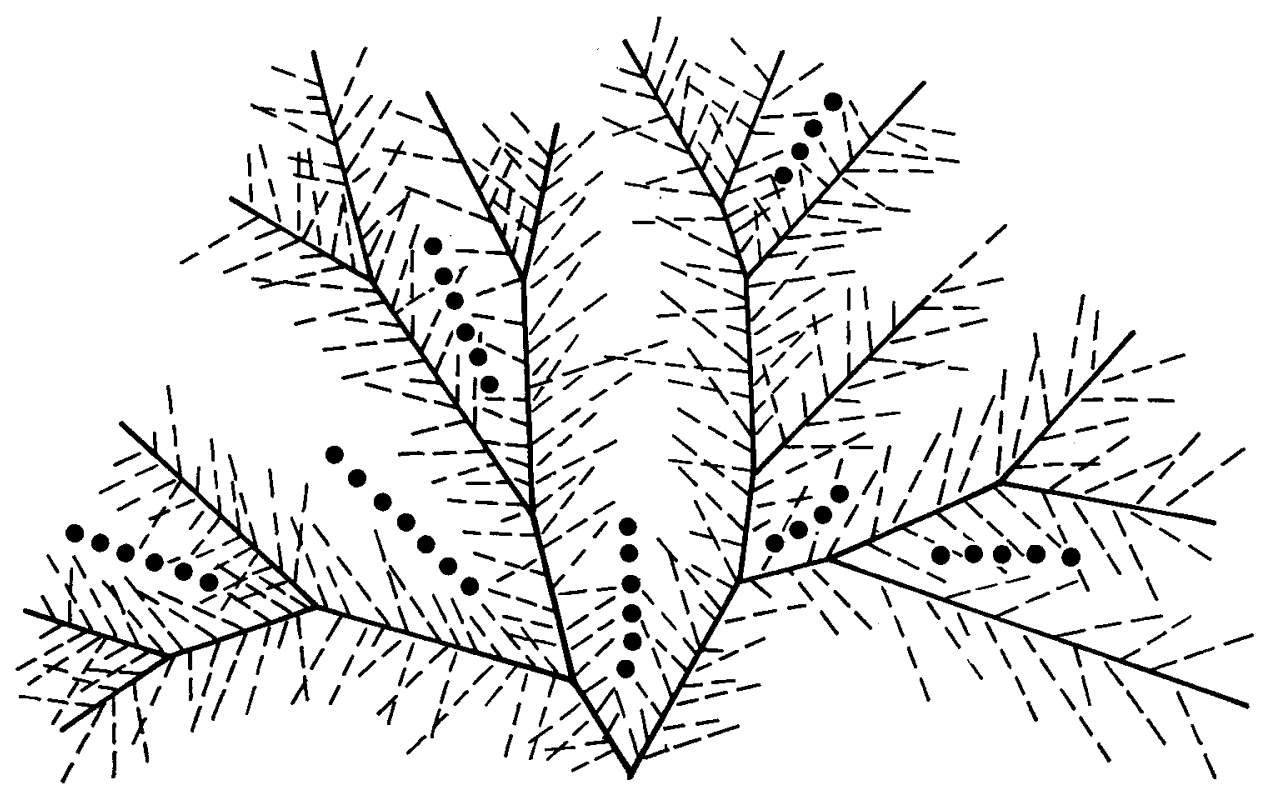

FIG. 1. Main lines represent normal diploid, sexual, outbreeding species; broken lines indicate polyploids, apomicts, and inbreeders; dots stand for hybrids.

of evolutionary noise is produced through the repeated origin of populations with deleteriously modified life cycles in which the advantages of diploidy, sexuality, outbreeding, and species purity are counteracted or cancelled. Such deviations persist for longer or shorter periods while the normal parental stock continues to evolve. The important evolution of plants has been accomplished by the basic lines shown in Fig. 1. We can expect to find and study all sorts of deviations such as those discussed, but the most significant research we can do is that focused on normal, divergent species.

\section{References}

Camp, W. H. and C. L. Gilly, 1943 - The structure and origin of species. Brittonia 4: 323-385. Constance, L., 1964 - Systematic Botany - an unending synthesis. Taxon 13: 257-273.

Evans, A. M., 1964 - Ameiotic alternation of generations: a new life cycle in the ferns. Science 143: 261-263.

Farrar, D. R. 1967 - Gametophytes of four tropical fern genera reproducing independently of their sporophytes in the southern Appalachians. Science 155: 1266-1267.

Foster, A. S. and E. M. Gifford JR., 1957 - Comparative Morphology of Vascular Plants. W. H. Freeman Co., San Francisco.

LEwis, H. 1969 - Evolutionary processes in the ecosystem. BioScience 19: 223-227.

Mirov, N. T. 1967 - The genus Pinus. Ronald Press Co., N.Y.

Mosquin, T., 1966 - Reproductive specialization as a factor in the evolution of the Canadian Flora. In Taylor, R. L. and R. A. Ludwig (eds.) "The Evolution of Canada's Flora": 43-65. Univ, of Toronto Press.

RiL.ex, R. and C. N. Law, 1965 - Genetic variation in chromosome pairing. Adv. Genet. 13: $57-114$.

Stebbins, G. L., 1950 - Variation and Evolution in Plants. Columbia Univ. Press.

--, 1969 - The significance of hybridization in plant taxonomy and evolution. Taxon 18: 26-35. 
TAYıon, T. M. C. 1967 - Mecodium wrightii in British Columbia and Alaska. Amer. Fern J. $57: 1-6$.

WAGNER, W. H. Jr. 1963 - Biosystematics and taxonomic categories in Lower Vascular Plants. Regnum Veg. 27: 63-71.

--, 1968a - Plant taxonomy and modern systematics. BioScience 18: 96-101.

-, $1968 \mathrm{~b}$ - Hybridization, taxonomy, and evolution. In Ileywood, V. H. "Modern Methods in Plant Taxonomy": 113-138. Acad. Press, London.

--, $1969-$ The role and taxonomic treatment of hybrids. BioScience 19: 785-789, 795. 